TITLE:

\title{
Ultrafast photodynamics of pyrazine in the vacuum ultraviolet region studied by time-resolved photoelectron imaging using 7.8-eV pulses
}

\section{AUTHOR(S):}

Horio, Takuya; Suzuki, Yoshi Ichi; Suzuki, Toshinori

\section{CITATION:}

Horio, Takuya ...[et al]. Ultrafast photodynamics of pyrazine in the vacuum ultraviolet region studied by time-resolved photoelectron imaging using 7.8-eV pulses. Journal of Chemical Physics 2016, 145(4): 044307.

\section{ISSUE DATE:}

2016-07-28

URL:

http://hdl.handle.net/2433/230214

\section{RIGHT:}

This article may be downloaded for personal use only. Any other use requires prior permission of the author and AIP Publishing. The following article appeared in 'The Journal of Chemical Physics 145, 044307 (2016)' and may be found at

https://aip.scitation.org/doi/10.1063/1.4955298.; The full-text file will be made open to the public on 28 July 2017 in accordance with publisher's 'Terms and Conditions for SelfArchiving' 


\section{Ultrafast photodynamics of pyrazine in the vacuum ultraviolet region studied by time-resolved photoelectron imaging using 7.8-eV pulses}

Takuya Horio, Yoshi-ichi Suzuki, and Toshinori Suzuki

Citation: The Journal of Chemical Physics 145, 044307 (2016); doi: 10.1063/1.4955298

View online: https://doi.org/10.1063/1.4955298

View Table of Contents: http://aip.scitation.org/toc/jcp/145/4

Published by the American Institute of Physics

\section{Articles you may be interested in}

Full observation of ultrafast cascaded radiationless transitions from $S_{2}(\pi \pi)$ state of pyrazine using vacuum ultraviolet photoelectron imaging

The Journal of Chemical Physics 145, 044306 (2016); 10.1063/1.4955296

Real-time detection of $S\left({ }^{1} D_{2}\right)$ photofragments produced from the ${ }^{1} B_{2}\left({ }^{1} \Sigma_{u}{ }^{+}\right)$state of $C S_{2}$ by vacuum ultraviolet photoelectron imaging using $133 \mathrm{~nm}$ probe pulses

The Journal of Chemical Physics 147, 013932 (2017); 10.1063/1.4982219

Excited-state dynamics of furan studied by sub-20-fs time-resolved photoelectron imaging using 159-nm pulses

The Journal of Chemical Physics 143, 014302 (2015); 10.1063/1.4922904

Velocity map imaging of ions and electrons using electrostatic lenses: Application in photoelectron and photofragment ion imaging of molecular oxygen

Review of Scientific Instruments 68, 3477 (1997); 10.1063/1.1148310

Observation of the wavepacket dynamics on the ${ }^{1} \mathrm{~B}_{2}\left({ }^{1} \Sigma_{\mathrm{u}}{ }^{+}\right)$state of $\mathrm{CS}_{2}$ by sub-20 fs photoelectron imaging using $159 \mathrm{~nm}$ probe pulses

The Journal of Chemical Physics 142, 074308 (2015); 10.1063/1.4907749

Time-resolved photoelectron imaging of ultrafast $S_{2} \rightarrow S_{1}$ internal conversion through conical intersection in pyrazine

The Journal of Chemical Physics 132, 174302 (2010); 10.1063/1.3395206

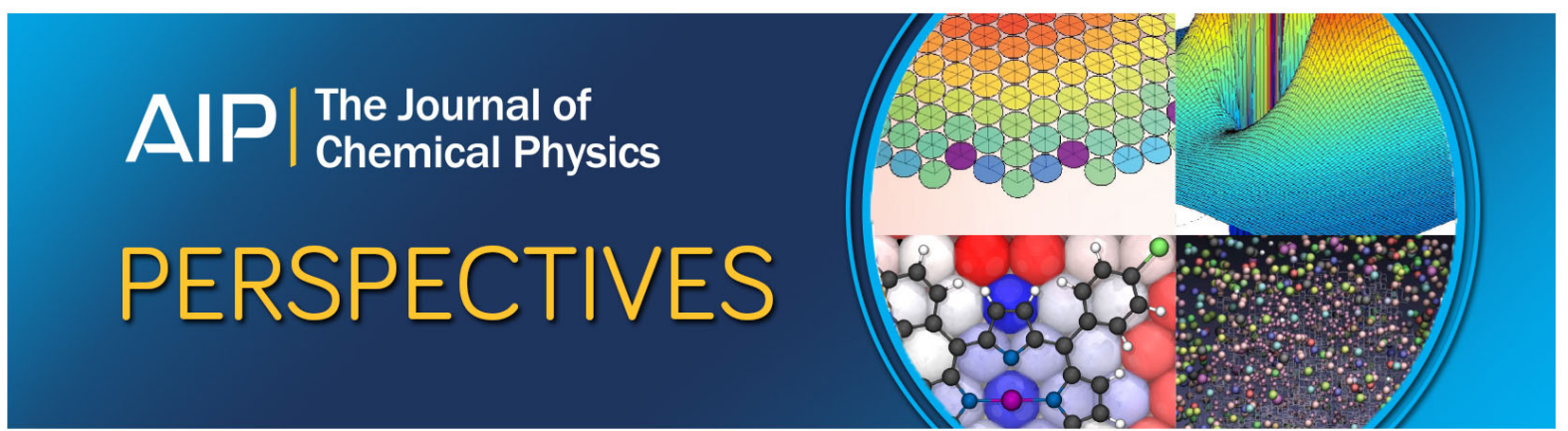




\title{
Ultrafast photodynamics of pyrazine in the vacuum ultraviolet region studied by time-resolved photoelectron imaging using 7.8-eV pulses
}

\author{
Takuya Horio, Yoshi-ichi Suzuki, a) and Toshinori Suzukib) \\ Department of Chemistry, Graduate School of Science, Kyoto University, Kitashirakawa Oiwake-cho, \\ Sakyo-Ku, Kyoto 606-8502, Japan
}

(Received 1 April 2016; accepted 20 May 2016; published online 25 July 2016)

\begin{abstract}
The ultrafast electronic dynamics of pyrazine $\left(\mathrm{C}_{4} \mathrm{~N}_{2} \mathrm{H}_{4}\right)$ were studied by time-resolved photoelectron imaging (TRPEI) using the third $(3 \omega, 4.7 \mathrm{eV})$ and fifth harmonics $(5 \omega, 7.8 \mathrm{eV})$ of a femtosecond Ti:sapphire laser $(\omega)$. Although the photoionization signals due to the $5 \omega-3 \omega$ and $3 \omega-5 \omega$ pulse sequences overlapped near the time origin, we have successfully extracted their individual TRPEI signals using least squares fitting of the observed electron kinetic energy distributions. When the $5 \omega$ pulses preceded the $3 \omega$ pulses, the $5 \omega$ pulses predominantly excited the $S_{4}\left(\pi \pi^{*},{ }^{1} B_{1 u}+{ }^{1} B_{2 u}\right)$ state. The photoionization signal from the $S_{4}$ state generated by the time-delayed $3 \omega$ pulses was dominated by the $D_{3}\left({ }^{2} B_{2 g}\right) \leftarrow S_{4}$ photoionization process and exhibited a broad electron kinetic energy distribution, which rapidly downshifted in energy within $100 \mathrm{fs}$. Also observed were the photoionization signals for the $3 s, 3 p_{z}$, and $3 p_{y}$ members of the Rydberg series converging to $D_{0}\left({ }^{2} A_{g}\right)$. The Rydberg signals appeared immediately within our instrumental time resolution of $27 \mathrm{fs}$, indicating that these states are directly photoexcited from the ground state or populated from $S_{4}$ within $27 \mathrm{fs}$. The $3 s, 3 p_{z}$, and $3 p_{y}$ states exhibited single exponential decay with lifetimes of $94 \pm 2,89 \pm 2$, and $58 \pm 1 \mathrm{fs}$, respectively. With the reverse pulse sequence of $3 \omega-5 \omega$, the ultrafast internal conversion (IC) from $S_{2}\left(\pi \pi^{*}\right)$ to $S_{1}\left(n \pi^{*}\right)$ was observed. The decay associated spectrum of $S_{2}$ exhibited multiple bands ascribed to $D_{0}, D_{1}$, and $D_{3}$, in agreement with the $3 \omega$-pump and $6 \omega$-probe experiment described in our preceding paper [T. Horio et al., J. Chem. Phys. 145, 044306 (2016)]. The electron kinetic energy and angular distributions from $S_{1}$ populated by IC from $S_{2}$ are also discussed. Published by AIP Publishing. [http://dx.doi.org/10.1063/1.4955298]
\end{abstract}

\section{INTRODUCTION}

In our preceding paper, ${ }^{30}$ we have discussed time-resolved photoelectron imaging (TRPEI) using the 6th harmonic ( $6 \omega$; $9.3 \mathrm{eV}$ ) of a femtosecond Ti:sapphire laser $(\omega)$ to fully probe cascaded radiationless transitions from an excited state. In this paper, we present TRPEI using the 5th harmonic $(5 \omega ; 7.8 \mathrm{eV})$ and discuss its characteristics in the studies of photophysics and photochemistry of molecules.

Figure 1 shows a photoabsorption spectrum of pyrazine $\left(\mathrm{C}_{4} \mathrm{~N}_{2} \mathrm{H}_{4}, D_{2 h}\right)$ vapor in the region from 4.5 to $12 \mathrm{eV}$. ${ }^{1}$ As seen here, vacuum UV (VUV) photoabsorption spectra of large molecules are highly congested, due to the large number of transitions to the Rydberg $(R)$ and higher-valence states $\left(S_{i}\right)$. However, it is noted that the broadness of the absorption bands is also indicative of the ultrafast dynamics of these states. The absorption cross sections are quite large in the VUV region; for example, the transition(s) to $S_{4}\left(\pi \pi^{*}\right)$, which corresponds to the ${ }^{1} E_{1 u}$ state of benzene $\left(D_{6 h}\right),{ }^{2}$ has a cross section as large as $120 \mathrm{Mb}$. A number of sharp peaks around the $S_{4}$ band are transitions to low-lying Rydberg states and associated vibrational structures. Because of such complex spectral features in the VUV region, photoexcitation in this

\footnotetext{
a)Present address: Faculty of Pharmaceutical Science, Health Sciences University of Hokkaido, Ishikari-Tobetsu, Hokkaido 061-0293, Japan.

b) Author to whom correspondence should be addressed. Electronic mail: suzuki@kuchem.kyoto-u.ac.jp
}

region is expected to initiate dynamics involving the strongly coupled valence and Rydberg states.

TRPEI is highly useful for studying complex ultrafast electronic dynamics. ${ }^{3}$ The method enables straightforward distinction of Rydberg states from higher-valence excited states, because photoionization from a Rydberg state provides a characteristic sharp photoelectron kinetic energy distribution (PKED), and valence states usually exhibit much broader PKEDs. Time-dependent photoelectron angular distributions (PADs) are also of great assistance for resolving these states, because Rydberg states generally exhibit strong photoemission anisotropy whereas valence states do not. While TRPEI studies in the VUV region have been scarce owing to the difficulty in generating ultrashort VUV pulses, filamentation four-wave mixing enables the generation of sub-20 fs VUV pulses using a relatively simple apparatus ${ }^{4,5}$ and has changed this situation.

In our previous study on VUV-TRPEI of furan $\left(\mathrm{C}_{4} \mathrm{OH}_{4}\right.$, $\left.C_{2 v}\right)$ using the 4 th harmonic $(4 \omega ; 6.3 \mathrm{eV})$ and $5 \omega$ pulses, ${ }^{6}$ a higher-valence ${ }^{1} A_{1}\left(\pi \pi^{*}\right)$ state was populated by the $5 \omega$ pulses, and an ultrafast ( $<100 \mathrm{fs})$ structural deformation on the ${ }^{1} A_{1}\left(\pi \pi^{*}\right)$ state was observed from the time-evolution of the PKEDs measured using the time-delayed $4 \omega$. One of the key findings was that low-lying Rydberg states, ${ }^{1} B_{1}\left(2 b_{1} \rightarrow 3 s\right),{ }^{1} B_{1}\left(1 a_{2} \rightarrow 3 p_{y}\right)$, and ${ }^{1} B_{2}\left(1 a_{2} \rightarrow 3 p_{x}\right)$, were populated via the ${ }^{1} A_{1}\left(\pi \pi^{*}\right)$ state, suggesting the existence of conical intersections (CIs) between the ${ }^{1} A_{1}\left(\pi \pi^{*}\right)$ state and 


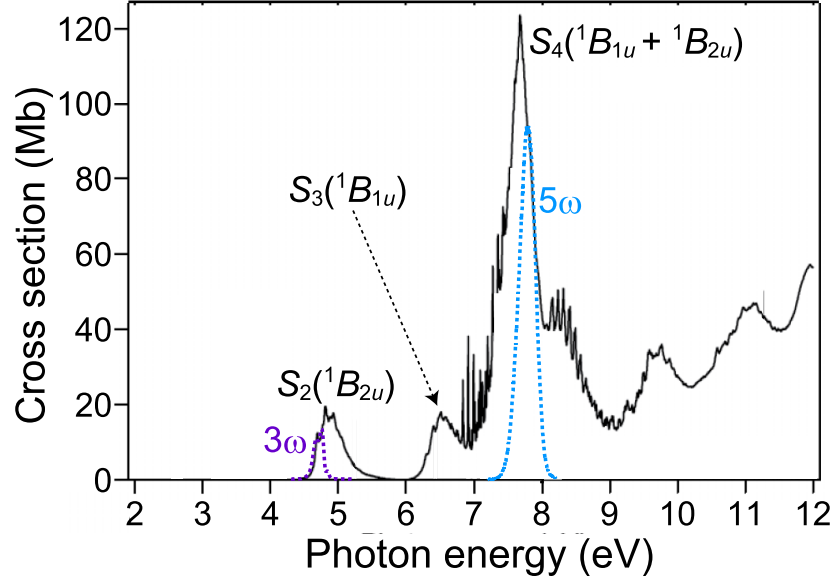

FIG. 1. Photoabsorption spectrum of pyrazine vapor from 4.5 to $12 \mathrm{eV}$ overlaid with the spectra of the third $(3 \omega, 4.7 \mathrm{eV})$ and fifth harmonics $(5 \omega$, $7.8 \mathrm{eV}$ ) pulses of a femtosecond Ti:sapphire laser $(\omega)$ used in this study. The photoabsorption spectrum was adapted with permission from M. Stener $e t$ al., J. Phys. B: At., Mol. Opt. 44, 075203 (2011). Copyright 2011 IOP Publishing Ltd.

the low-lying Rydberg states along the structural deformation coordinate.

In the present paper, we report similar VUV-TRPEI of pyrazine using $5 \omega$-pump and 3rd-harmonic $(3 \omega ; 4.7 \mathrm{eV})$ probe pulses, in order to further investigate the photophysics of large molecules in the VUV region. As shown in Fig. 1, 5w and $3 \omega$ are, respectively, resonant with transitions to $S_{4}$ and Rydberg states and with the $S_{2}\left(\pi \pi^{*},{ }^{1} B_{2 u}\right) \leftarrow S_{0}\left({ }^{1} A_{g}\right)$ transition. As mentioned in our preceding paper, ${ }^{30}$ the $S_{2}\left(\pi \pi^{*}\right) \rightarrow S_{1}\left(n \pi^{*}\right)$ internal conversion (IC) in pyrazine is a benchmark system to study ultrafast non-adiabatic transitions via a CI of potential energy surfaces (PESs), which motivated us to employ $3 \omega$ in combination with $5 \omega$ in the present study; the reverse pulse sequence, i.e., a $3 \omega$-pump and $5 \omega$-probe scheme, enabled us to explore this benchmark IC process. As reported in the preceding paper ${ }^{30}$ the ultrafast cascaded radiationless transitions from $S_{2}\left(\pi \pi^{*}\right)$ of pyrazine were fully observed by TRPEI using $6 \omega$. The TRPEI experiment on pyrazine using $5 \omega$ presented here was performed prior to our $6 \omega$ experiment, and the results motivated us to extend it to $6 \omega$. Both the $5 \omega-3 \omega$ and $3 \omega-5 \omega$ pulse sequences create photoionization signals in the same energy region, and the photoionization signals overlap with each other around the time-origin. We will describe how these signals are deconvoluted to extract individual signals, discuss the characteristics of TRPEI using $5 \omega$, and compare the results with those of TRPEI using $6 \omega$.

\section{EXPERIMENTAL METHOD}

The light source, photoelectron imaging apparatus, data acquisition system, and image analysis procedures used in this study were identical to those described in the preceding paper. ${ }^{30}$ The pump-probe setup using $3 \omega$ and $5 \omega$ pulses was realized by replacing the dielectric mirrors designed for $6 \omega$ used in the preceding study with those for $5 \omega$. The pulse energies of $3 \omega$ and $5 \omega$ on the molecular beam were estimated to be $\sim 500 \mathrm{~nJ} /$ pulse and $\sim 50 \mathrm{~nJ} / \mathrm{pulse}$, respectively. The cross-correlation function between the $3 \omega$ and $5 \omega$ pulses was measured using non-resonant $\left(1+1^{\prime}\right)$ multiphoton ionization of xenon, and it was found to be a single Gaussian with a full width at half maximum (FWHM) of 27 fs. Figure 2 shows a schematic diagram of the pump-probe scheme employed in this study. For positive delay times, $5 \omega$ preceded $3 \omega$, so that $5 \omega$ and $3 \omega$ acted as the pump and probe pulses, respectively, and vice versa for negative delay times. Photoelectron images were measured as a function of the pump-probe delay time from -162 to 183 fs at 5 fs intervals. Each image was integrated for $13 \mathrm{~s}$, and the measurement was repeated 30 times. The 30 images at each delay time were combined, from which the original three-dimensional (3D) scattering distributions were reconstructed using $p$-BASEX. ${ }^{7}$ Time-independent background images due to one-color photoionization by each pulse were subtracted prior to the reconstruction. PKE calibration was performed using one-color three-photon ionization of xenon with $3 \omega$ pulses.

We employed $\left(1+1^{\prime}\right)$ resonantly enhanced multiphoton ionization (REMPI) using linearly polarized pump and probe pulses with parallel polarizations. Therefore, the timedependent photoionization cross sections, $I(E, \theta, t)$, can be expressed as

$I(E, \theta, t)=\frac{\sigma(E, t)}{4 \pi}\left[1+\beta_{2}(E, t) P_{2}(\cos \theta)+\beta_{4}(E, t) P_{4}(\cos \theta)\right]$,

where $E, \theta$, and $t$, respectively, denote the PKE, photoelectron ejection angle with respect to the laser polarization and pump-probe delay time, and $P_{n}(x)$ is an $n$th order Legendre polynomial. In addition, $\sigma(E, t)$ denotes the PKEDs, and the coefficients, $\beta_{2}$ and $\beta_{4}$, are photoelectron anisotropy parameters that depend on both $E$ and $t$.

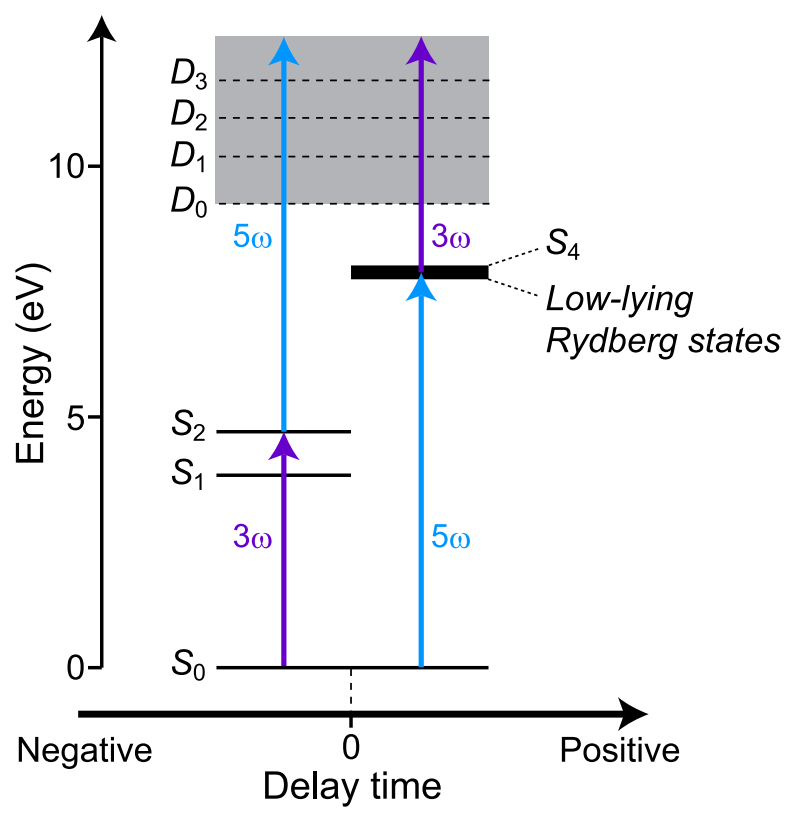

FIG. 2. Schematic diagram of the pump-probe scheme employed in this study. 


\section{RESULTS AND DISCUSSION}

Figure 3 shows two-dimensional (2D) slices through the $3 \mathrm{D}$ photoelectron scattering distributions at delay times of -22 and $38 \mathrm{fs}$, which highlights that entirely different distributions occur between the $5 \omega-3 \omega$ and $3 \omega-5 \omega$ pulse sequences.

The total photoelectron yield is presented in Fig. 4(a) as a function of the delay time between the $3 \omega$ and $5 \omega$ pulses. In the plot, the time-independent background signals due to one-color photoionizations have been subtracted so that zero intensity corresponds to the signal level of the time-independent one-color background. Time-dependent photoionization signals can be seen for both the $5 \omega-3 \omega$ and $3 \omega-5 \omega$ pulse sequences. In Subsections III A and III B, the results observed for these pulse sequences are discussed separately.

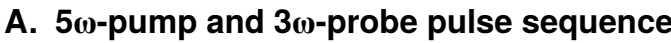

Figure 4(b) shows a 2D time-energy map of PKEDs, namely, $\sigma(E, t)$ in Eq. (1). The map exhibits a short-lived component with a broad energy distribution in the PKE region lower than $\sim 1 \mathrm{eV}$, and three long-lived components in the PKE region between 1.4 and $3 \mathrm{eV}$, whose characteristic feature is their narrow bandwidth. As discussed in Section I, the broad and sharp components of $\sigma(E, t)$ can be immediately assigned to photoionization from valence-excited and Rydberg states, respectively. These assignments are further supported by examining the 2D map of $\beta_{2}(E, t)$ presented in Fig. 4(d). It is clear from the figure that $\beta_{2}(E, t)$ is strongly dependent on $E$ : the $\beta_{2}$ values in the PKE regions where the three sharp components (Rydberg states) were observed are quite high $(0.8-1.1)$, as denoted using red, while the broad component from the valence state exhibits much lower $\beta_{2}$ values (0.0-0.4). These are well-established fingerprints for photoionization processes from Rydberg and valence states. The results clearly demonstrate that 2D mapping of $\sigma(E, t)$ and $\beta_{2}(E, t)$ by TRPEI is effective for discerning the electronic characters of non-stationary states in the VUV region.

Let us first examine the broad PKED in Fig. 4(b). Based on Fig. 1, this broad component is unambiguously assigned to photoionization from the $S_{4}\left(\pi \pi^{*}\right)$ state created by the $5 \omega$ pulses. Theoretical investigations on the electronic excited states of pyrazine ${ }^{8-11}$ showed that the electronic character

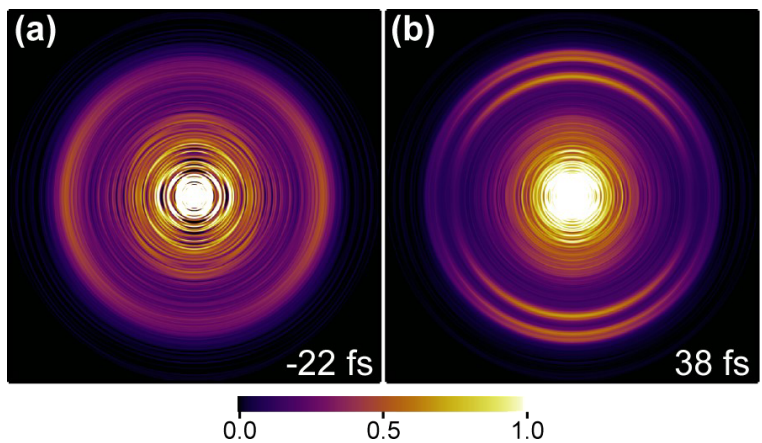

FIG. 3. 2D slices of the 3D photoelectron scattering distributions obtained at the delay times of (a) -22 and (b) $38 \mathrm{fs}$.
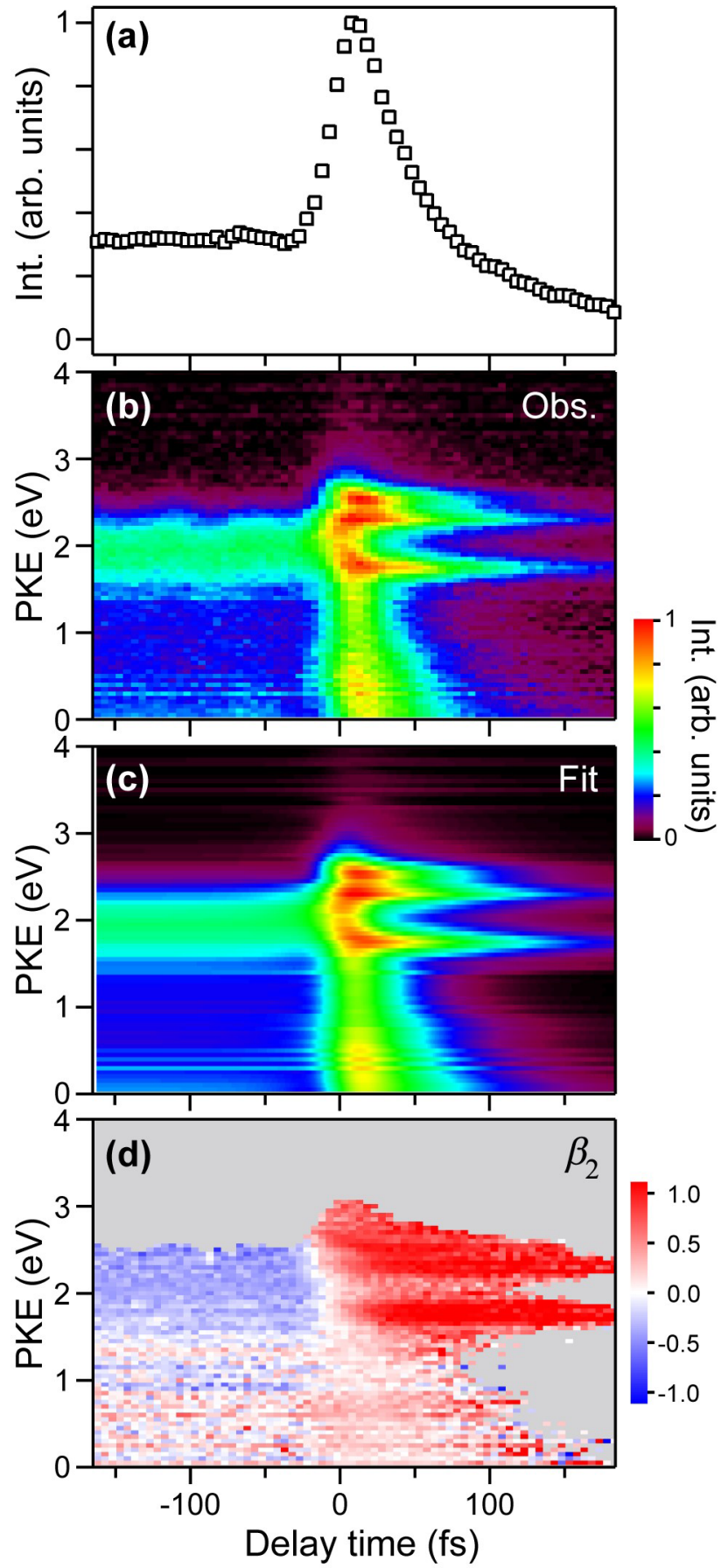

FIG. 4. (a) Total photoelectron yield as a function of the delay time between the $3 \omega$ and $5 \omega$ pulses. For positive delay times, $5 \omega$ preceded $3 \omega$, and vice versa for negative delay times. (b) Observed and (c) fitting result of timedependent photoelectron kinetic energy distributions. (d) Time-dependent photoelectron anisotropy parameter, $\beta_{2}$. The $\beta_{2}$ values are displayed only in the regions where the normalized photoelectron intensities in (b) are greater than 0.1 .

of the $S_{4}\left(\pi \pi^{*}\right)$ state can be described as ${ }^{1} B_{1 u}+{ }^{1} B_{2 u}$, as they are almost degenerate. The former $\left({ }^{1} B_{1 u}\right)$ has a leading configuration of $\left(1 b_{2 g}\right)^{-1}\left(2 b_{3 u}\right)^{+1}$, while the latter $\left({ }^{1} B_{2 u}\right)$ has a leading configuration of $\left(1 b_{2 g}\right)^{-1}\left(1 a_{u}\right)^{+1}$. Since $D_{3}\left({ }^{2} B_{2 g}\right)$ has a single leading configuration of $\left(1 b_{2 g}\right)^{-1},{ }^{12-14}$ both of the photoionization processes, $\left(1 b_{2 g}\right)^{-1} \leftarrow\left(1 b_{2 g}\right)^{-1}\left(2 b_{3 u}\right)^{+1}$ and $\left(1 b_{2 g}\right)^{-1} \leftarrow\left(1 b_{2 g}\right)^{-1}\left(1 a_{u}\right)^{+1}$, are allowed: an electron in the $2 b_{3 u}$ or the $1 a_{u}$ orbital is ejected by the $3 \omega$ pulses 
without changing the electronic configuration of the ion core, $\left(1 b_{2 g}\right)^{-1}$. Thus, photoionization from $S_{4}$ by the $3 \omega$ probe pulses predominantly yields $D_{3}$. In the present study, the total photon energy of $3 \omega$ and $5 \omega$ is $12.5 \mathrm{eV}$, indicating that the four cationic states $D_{0}\left({ }^{2} A_{g}\right), D_{1}\left({ }^{2} B_{1 g}\right), D_{2}\left({ }^{2} B_{1 \mathrm{u}}\right)$, and $D_{3}\left({ }^{2} B_{2 g}\right)$ are energetically accessible. The largest possible PKEs, $E_{i}^{\text {max }}$, were estimated for each cationic state, $D_{i}$, from high-resolution photoelectron spectra ${ }^{15,16}$ in the literature. The estimated values for $E_{0}^{\max }, E_{1}^{\max }, E_{2}^{\max }$, and $E_{3}^{\max }$ are $3.21,2.33,1.57$, and $0.84 \mathrm{eV}$, respectively, suggesting that the broad component in the lower energy region $(<1 \mathrm{eV})$ should be assigned to $D_{3} \leftarrow S_{4}$.

As can be seen in Fig. 4(b), the $S_{4}$ PKED exhibits a progressive energy shift towards zero PKE, indicating wavepacket motion on the $S_{4}$ PES. When the wavepacket moves away from the Franck-Condon region, higher photon energy is required for photoionization: The photon energy of the $3 \omega$ pulse $(4.7 \mathrm{eV})$ is insufficient to induce ionization from the entire $S_{4} \mathrm{PES}$, leading to diminished photoelectron intensity. Thus, the photoelectron intensity does not represent the $S_{4}$ population accurately. The wavepacket motion can be related to some ultrafast structural deformation occurring on the $S_{4}$ state, although the deformation coordinate is unclear at this point.

Next, we discuss the Rydberg states observed as three sharp bands in the PKE region from 1.4 to $3.0 \mathrm{eV}$. For a closer examination of the three components, Fig. 5 presents PKEDs obtained at 28, 43, 58, and $118 \mathrm{fs}$. The three dotted lines in the figure indicate their peak positions. The term values of the observed Rydberg states, $T$, can be calculated using the following relation:

$$
T=P K E+I E-\hbar \omega_{\text {probe }},
$$

where $I E$ and $\hbar \omega_{\text {probe }}$ are the ionization energy of pyrazine for $D_{0}\left(n^{-1},{ }^{2} A_{g}\right)(9.288 \mathrm{eV})^{16}$ and the probe photon energy, respectively. The term values thus calculated are summarized in Table I.

Walker and Palmer ${ }^{9}$ estimated the term values of the $3 p_{x}\left(n^{-1}\right), 3 p_{y}\left(n^{-1}\right)$, and $3 p_{z}\left(n^{-1}\right)$ Rydberg states of pyrazine to be $6.75,7.07$, and $6.84 \mathrm{eV}$, respectively, using VUV photoabsorption spectroscopy, electron energy loss spectroscopy, and $a b$ initio multireference configuration interaction calculations. In our previous study using picosecond $(2+1)$ REMPI spectroscopy, ${ }^{16}$ we estimated the term value of the $3 s\left(n^{-1}\right)$ state of pyrazine to be $50844(8) \mathrm{cm}^{-1}(6.30 \mathrm{eV})$, which was

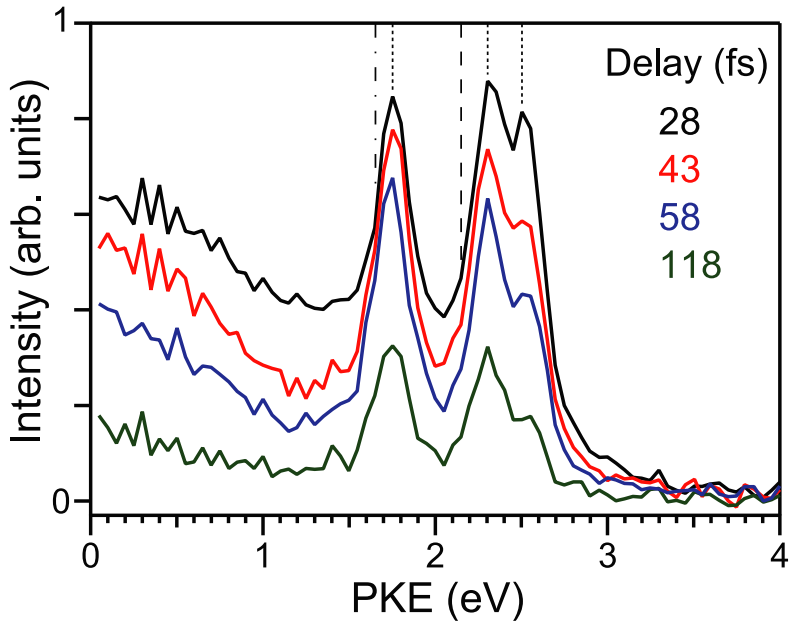

FIG. 5. PKEDs obtained at $28,43,58$, and 118 fs. Dotted lines indicate the peak positions for ${ }^{1} R(3 s),{ }^{1} R\left(3 p_{z}\right)$, and ${ }^{1} R\left(3 p_{y}\right)$ (see the text). The expected PKEs for the ${ }^{1} R\left(3 p_{x}\right)$ and $3 \mathrm{~s}\left(\pi^{-1}\right)$ Rydberg states are shown using dashed and dashed-dotted lines, respectively.

in fair agreement with the estimated values of $50830 \mathrm{~cm}^{-1}$ by Turner et al. ${ }^{17}$ and $50835 \mathrm{~cm}^{-1}$ by Dion and Bernstein ${ }^{18}$ using nanosecond $(2+1)$ REMPI spectroscopy. Using femtosecond $(2+1)$ REMPI spectroscopy, Song et al. ${ }^{19}$ measured the term values of $3 p_{y}\left(n^{-1}\right)$ and $3 p_{z}\left(n^{-1}\right)$ as $56800 \mathrm{~cm}^{-1}$ $(7.04 \mathrm{eV})$ and $55000 \mathrm{~cm}^{-1}(6.82 \mathrm{eV})$, respectively. Based on a comparison with the results of these previous studies, the three peaks shown in Fig. 5 are assigned to $3 s\left(n^{-1}\right)$, $3 p_{z}\left(n^{-1}\right)$, and $3 p_{y}\left(n^{-1}\right)$ in order of increasing PKE. No signal was observed for the $3 p_{x}\left(n^{-1}\right)$ Rydberg state, for which the expected PKE is $2.16 \mathrm{eV}$, as indicated by the dashed line in Fig. 5. In a previous study, ${ }^{19}$ we identified the $3 s\left(\pi^{-1}\right)$ Rydberg state with the term value of $7.130 \mathrm{eV}$. Based on this term value and the ionization energy of $D_{1}$ $\left(\pi^{-1}\right), 10.169 \mathrm{eV}$, its signal for the current pump-probe scheme is expected at $1.657 \mathrm{eV}$, as indicated by the dasheddotted line in Fig. 5. However, no noticeable signal of the $3 \mathrm{~s}\left(\pi^{-1}\right)$ Rydberg state was identified. Hereafter, we denote the $3 s\left(n^{-1}\right), 3 p_{x}\left(n^{-1}\right), 3 p_{y}\left(n^{-1}\right)$, and $3 p_{z}\left(n^{-1}\right)$ Rydberg states as ${ }^{1} R(3 s),{ }^{1} R\left(3 p_{x}\right),{ }^{1} R\left(3 p_{y}\right)$, and ${ }^{1} R\left(3 p_{z}\right)$, as in the preceding paper. $^{30}$

To evaluate the lifetimes of the ${ }^{1} R(3 s),{ }^{1} R\left(3 p_{z}\right)$, and ${ }^{1} R\left(3 p_{y}\right)$ states, we extracted their photoelectron signals in the PKE regions of $1.70-1.80,2.25-2.35$, and $2.45-2.55 \mathrm{eV}$

TABLE I. Summary of the observed Rydberg states. Term values from the literature are also shown. Lifetimes were obtained using Eq. (3).

\begin{tabular}{lcccccc}
\hline \hline & \multicolumn{6}{c}{ Term values (eV) } \\
Position (eV) & This work & References $16-18$ & Reference 9 & Reference 19 & Assignment $^{\mathrm{a}}$ & Lifetime (fs) $^{\mathrm{b}}$ \\
\hline 2.50 & 7.09 & $\ldots$ & $7.07(7.20)^{\mathrm{c}}$ & 7.04 & $3 p_{y}\left(n^{-1}\right),{ }^{1} B_{2 u}$ & $58 \pm 1$ \\
2.30 & 6.89 & $\ldots$ & $6.84(6.94)^{\mathrm{c}}$ & 6.82 & $3 p_{z}\left(n^{-1}\right),{ }^{1} B_{1 u}$ & $89 \pm 2$ \\
1.75 & 6.34 & 6.30 & $(6.11)^{\mathrm{c}}$ & 6.29 & $3 s\left(n^{-1}\right),{ }^{1} A_{g}$ & $94 \pm 2$ \\
\hline \hline
\end{tabular}

a The $z$-axis is defined along the $C_{2}$ axis through the two nitrogen atoms, and the $x$-axis is perpendicular to the molecular plane.

${ }^{\mathrm{b}}$ Note that these lifetimes were obtained for $3 p_{y}\left(n^{-1}\right), 3 p_{z}\left(n^{-1}\right)$, and $3 s\left(n^{-1}\right)$ Rydberg states with internal energies of $0.71,0.91$, and $1.46 \mathrm{eV}$, respectively.

${ }^{\mathrm{c}}$ The values in parentheses were obtained by ab initio multireference configuration interaction calculations. 
and performed least squares fitting using the following formula:

$$
\begin{aligned}
I(t)= & {\left[C_{R} \exp \left(-\frac{t}{\tau_{R}}\right)+C_{S_{4}} \exp \left(-\frac{t}{\tau_{S_{4}}}\right)+C_{S_{2}} \exp \left(\frac{t}{\tau_{S_{2}}}\right)\right.} \\
& \left.+C_{S_{1}}\left(1-\exp \left(\frac{t}{\tau_{S_{2}}}\right)\right)\right] \otimes g(t),
\end{aligned}
$$

where $g(t)$ is the pump-probe cross-correlation function. $C_{R} / C_{S_{4}}$ and $\tau_{R} / \tau_{S_{4}}$, respectively, denote the amplitudes of the Rydberg $/ S_{4}$ component and their time constants. The ultrafast IC process from $S_{2}$ to $S_{1}$ observed at negative time delays was also taken into account. The $S_{2}$ lifetime is $22 \mathrm{fs},{ }^{20}$ while $S_{1}$ populated via IC from $S_{2}$ is rather long-lived. Therefore, the photoionization signal from $S_{1}$ yields the plateau visible in Fig. 4(a). In the least squares fitting, we fixed the $S_{2}$ lifetime, $\tau_{S 2}$, to be $22 \mathrm{fs}$.

The extracted photoionization signals and the results of the least squares fitting are presented in Fig. 6. The least squares fitting well reproduces the transient signals for both positive and negative time delays. The lifetimes of ${ }^{1} R(3 s)$, ${ }^{1} R\left(3 p_{z}\right)$, and ${ }^{1} R\left(3 p_{y}\right)$ are summarized in Table I. Note that our excitation pulses create ${ }^{1} R(3 s),{ }^{1} R\left(3 p_{z}\right)$, and ${ }^{1} R\left(3 p_{y}\right)$ with excess energies of 1.46, 0.91, and $0.71 \mathrm{eV}$, respectively; therefore, lifetimes at their origin (zero vibrational level in each state) are expected to be longer than the values observed in the present study. In fact, the lifetime for ${ }^{1} R(3 s)$ at the origin has been estimated to be $300 \mathrm{fs}$ by Dion and Bernstein. ${ }^{18}$

In our previous TRPEI study on furan using $4 \omega$ and $5 \omega$ pulses, ${ }^{6}$ a higher-valence ${ }^{1} A_{1}\left(\pi \pi^{*}\right)$ state was populated by the $5 \omega$ pump pulses, and its ultrafast dynamics were clearly observed by one-photon ionization using the $4 \omega$ probe pulses. The 2D map of the observed PKEDs was dominated by multiple photoionization signals from ${ }^{1} A_{1}\left(\pi \pi^{*}\right)$ into $D_{0}, D_{1}$, and $D_{2}$. Three low-lying Rydberg states, ${ }^{1} B_{1}\left(2 b_{1} \rightarrow 3 s\right),{ }^{1} B_{1}\left(1 a_{2} \rightarrow 3 p_{y}\right)$, and ${ }^{1} B_{2}\left(1 a_{2} \rightarrow 3 p_{x}\right)$, were also identified in the $2 \mathrm{D}$ map. Interestingly, the three Rydberg states appeared with time delays (9, 28, and
18 fs for ${ }^{1} B_{1}\left(2 b_{1} \rightarrow 3 s\right), \quad{ }^{1} B_{1}\left(1 a_{2} \rightarrow 3 p_{y}\right)$, and ${ }^{1} B_{2}\left(1 a_{2}\right.$ $\left.\rightarrow 3 p_{x}\right)$, respectively), indicating that they were populated via ${ }^{1} A_{1}\left(\pi \pi^{*}\right)$. However, such delayed appearances of the Rydberg states were not identified in the present study. This indicates that the ${ }^{1} R(3 s),{ }^{1} R\left(3 p_{z}\right)$, and ${ }^{1} R\left(3 p_{y}\right)$ Rydberg states observed in the present study were directly populated by the $5 \omega$ pump pulses. However, an inconsistency with this interpretation is that the photoionization signals from the Rydberg states seem too strong in comparison with their weak photoabsorption features, as seen in Fig. 1. We therefore conjecture that the ${ }^{1} R(3 s),{ }^{1} R\left(3 p_{z}\right)$, and ${ }^{1} R\left(3 p_{y}\right)$ Rydberg states observed in the present study were populated from the $S_{4}$ state in less than our instrumental time resolution (27 fs).

\section{B. 3w-pump and 5w-probe pulse sequence}

As shown in Fig. 1, $3 \omega$ is resonant with the $S_{2}\left(\pi \pi^{*},{ }^{1} B_{2 u}\right) \leftarrow S_{0}\left({ }^{1} A_{g}\right)$ transition, so we were able to explore the $S_{2}\left(\pi \pi^{*},{ }^{1} B_{2 u}\right) / S_{1}\left(n \pi^{*},{ }^{1} B_{3 u}\right)$ IC process using a $3 \omega$-pump and $5 \omega$-probe pulse sequence. $S_{2}\left(\pi \pi^{*},{ }^{1} B_{2 u}\right)$ is extremely shortlived ( $22 \mathrm{fs}$ ), ${ }^{20}$ because the $S_{2} / S_{1}$ CI occurs in the vicinity of the Franck-Condon region. ${ }^{21}$ Consequently, photoionization signal from $S_{2}\left(\pi \pi^{*},{ }_{1}^{1} B_{2 u}\right)$ appears only around the time origin of the pump-probe delay time. Compared with our previous study using $4 \omega(6.3 \mathrm{eV})$ probe pulses ${ }^{20} 5 \omega(7.8 \mathrm{eV})$ enables photoionization from much wider regions of the $S_{2}$ and $S_{1}$ PESs. However, since the $5 \omega$-pump and $3 \omega$-probe pulse sequence creates photoionization signals in the same energy region, the photoionization signals around the time origin become highly congested, as can be seen in Fig. 4(b). In this study, we performed a global fitting by dividing the PKE region into two, as explained below.

As is clear from Figs. 4(b) and 5, no appreciable contributions from any Rydberg states were observed in the PKE regions of $0-1.40$ and $3.20-4.00 \mathrm{eV}$. Thus, we divided the observed 2D map of PKEDs in Fig. 4(b), $I(E, t)$, as follows:

$$
\begin{aligned}
I(E, t)= & {\left[C_{\text {short }}(E) \exp \left(-\frac{t}{\tau_{\text {short }(E)}}\right)+C_{S_{2}}(E) \exp \left(\frac{t}{\tau_{S_{2}}}\right)+C_{S_{1}}(E)\left(1-\exp \left(\frac{t}{\tau_{S_{2}}}\right)\right)\right] \otimes g(t), } \\
& (0<E \leq 1.40 \mathrm{eV}, 3.20<E \leq 4.00 \mathrm{eV}), \\
I(E, t)= & {\left[C_{\text {short }}(E) \exp \left(-\frac{t}{\tau_{\text {short }(E)}}\right)+C_{\text {long }}(E) \exp \left(-\frac{t}{\tau_{\text {long }(E)}}\right)+C_{S_{2}}(E) \exp \left(\frac{t}{\tau_{S_{2}}}\right)+C_{S_{1}}(E)\left(1-\exp \left(\frac{t}{\tau_{S_{2}}}\right)\right)\right] \otimes g(t), }
\end{aligned}
$$

$(1.40<E \leq 3.20 \mathrm{eV})$.

The short and long-lived components correspond to the $S_{4}$ and Rydberg states, respectively. However, it should be noted that $\tau_{\text {short }}(E)$ does not provide the lifetime for $S_{4}$ because the corresponding PKED clearly varies with the delay time due to the wavepacket motion on the PES, as previously mentioned. In this sense, $\tau_{\text {short }}(E)$ is a PKE- dependent phenomenological time constant used to describe the passage of a wavepacket. We extracted the time profiles for each PKE bin with a $0.05-\mathrm{eV}$ width and performed least squares fitting to determine the $C_{S_{2}}(E)$ and $C_{S_{1}}(E)$ coefficients, which provide the PKEDs for $S_{2}$ and $S_{1}$, respectively. The result of the least squares fitting is presented as a $2 \mathrm{D}$ 


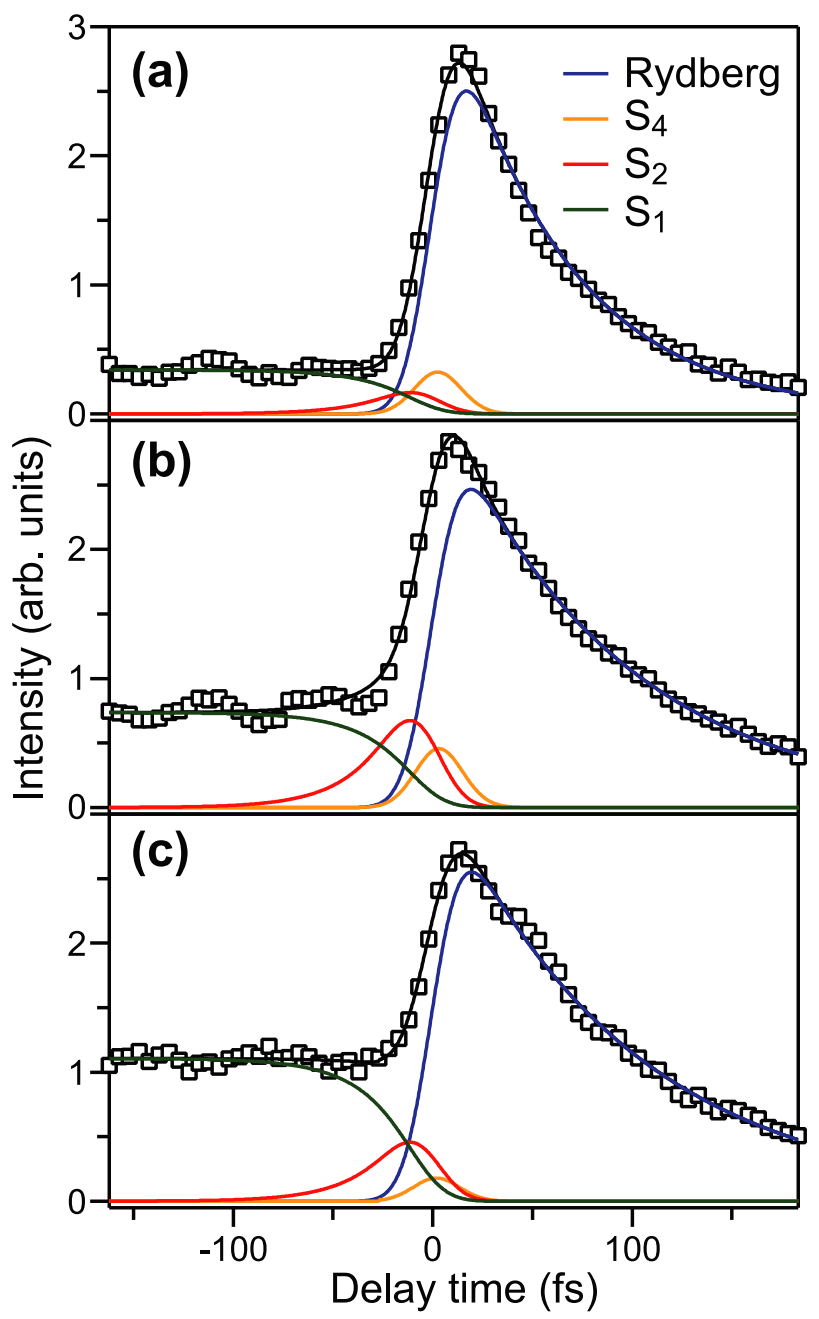

FIG. 6. Photoelectron time profiles obtained for the PKE subsections: (a) $2.45-2.55$, (b) $2.25-2.35$, and (c) $1.70-1.80 \mathrm{eV}$. The results of the least squares fittings are also shown with solid lines.

map in Fig. 4(c), which well reproduces the observed 2D map.

The coefficients $C_{S_{2}}(E)$ and $C_{S_{1}}(E)$ thus determined are presented in Figs. 7(a) and 7(b), respectively. For $C_{S_{2}}(E)$, two bands are clearly discernible at $\sim 0.6$ and $2.1 \mathrm{eV}$. The aforementioned values of $E_{1}^{\max }$ and $E_{3}^{\max }$ are presented with dotted lines in Fig. 7(a), which indicate that the two bands at $\sim 0.6$ and $2.1 \mathrm{eV}$ originate from $D_{3} \leftarrow S_{2}$ and $D_{1} \leftarrow S_{2}$ photoionization processes, respectively. A small intensity on the high energy side $\left(>2.5 \mathrm{eV}\right.$ ) of the $D_{1}$ band is ascribed to $D_{0} \leftarrow S_{2}$ photoionization.

Figure 8 compares the photoelectron spectra for $S_{2}$ obtained by the $3 \omega-5 \omega$ (this study) and $3 \omega-6 \omega$ (the preceding paper $^{30}$ ) experiments, which are presented as a function of electron binding energy (eBE). The results are consistent in terms of the band positions. Note that the $D_{x} \leftarrow S_{2}$ photoionization process, which was observed in the $3 \omega-6 \omega$ experiment (see Figs. 5(a) and 5(b) in the preceding paper $^{30}$ ), could not be observed in this study due to insufficient photon energy of the $5 \omega$ probe pulse. There are two noticeable changes when the probe pulse is switched from $6 \omega$ to $5 \omega$. One is the relative intensity of the $D_{3}$ band with respect to that of $D_{1}$, and the other is the contribution of the $D_{0}$ band.

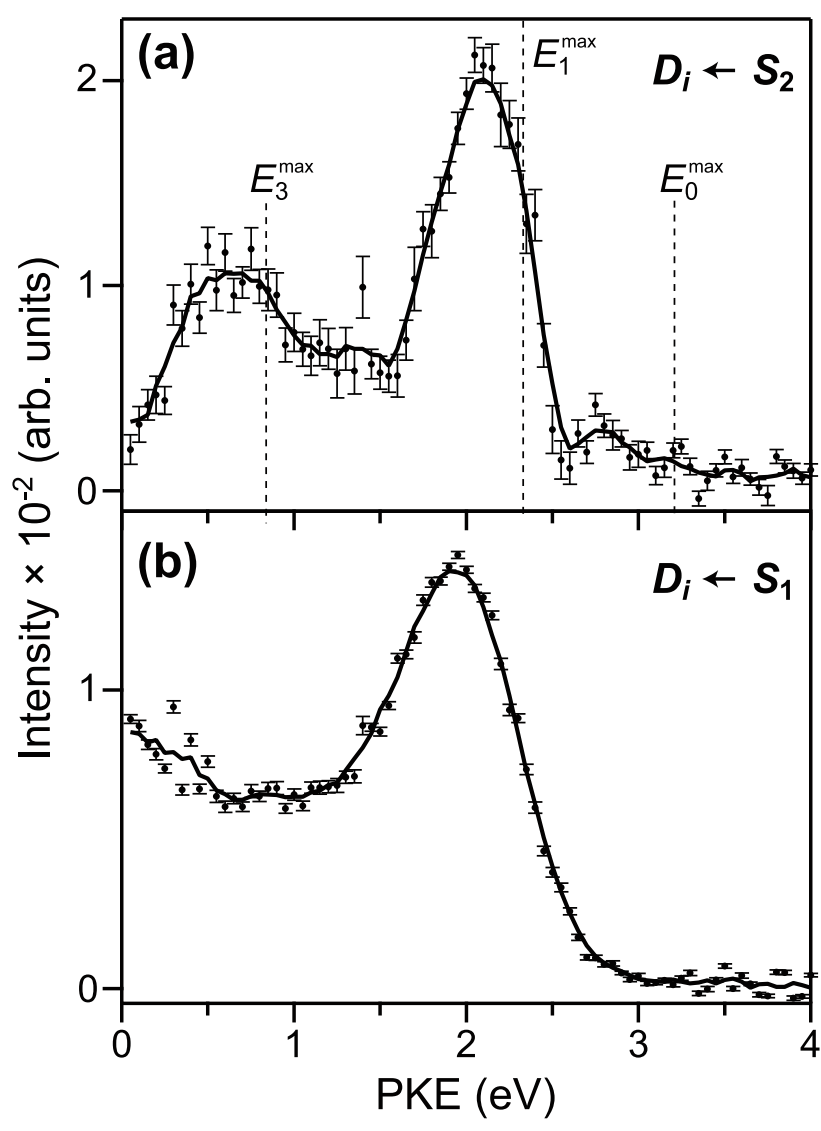

FIG. 7. The coefficients (a) $C_{S_{2}}(E)$ and (b) $C_{S_{1}}(E)$ obtained from the fitting result shown in Fig. 4(c) (filled circles). The error bars correspond to one standard deviation in the fit. The results obtained by 5-points box smoothing (moving average) are presented with solid curves.

These changes suggest that the relative magnitudes of the photoionization cross sections from $S_{2}$ into $D_{0}, D_{1}$, and $D_{3}$ are PKE-dependent. As for the lower intensity of the $D_{0}$ band in the $5 \omega$ experiment, it is possible that interference from the intense signals from the ${ }^{1} R\left(3 p_{z}\right)$ and ${ }^{1} R\left(3 p_{y}\right)$ Rydberg states

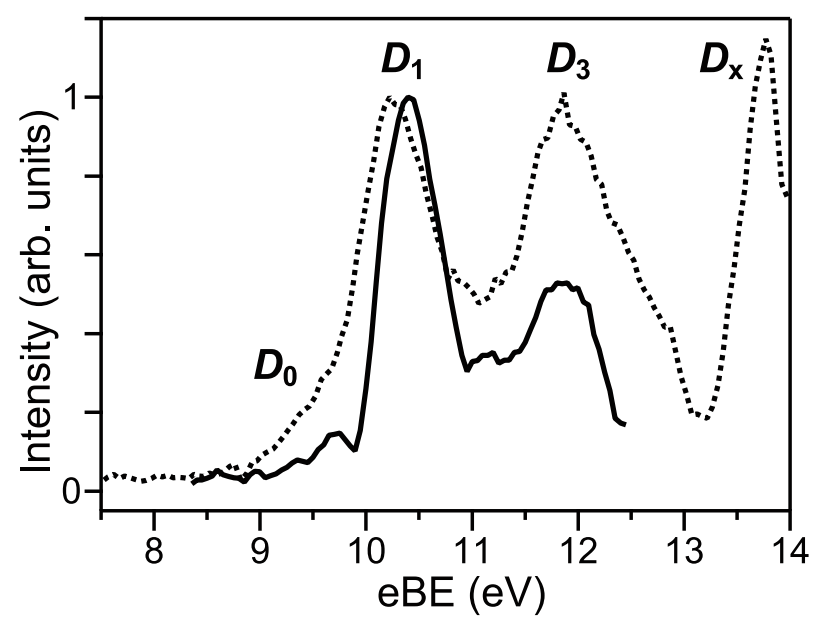

FIG. 8. Comparison of the photoelectron spectra for $S_{2}$ obtained by $5 \omega$ (solid line) and $6 \omega$ (dotted line) probe pulses. The solid line corresponds to the smoothed one shown in Fig. 7(a), while the dotted line was taken from the data shown in Fig. 5(a) of the preceding paper. ${ }^{30}$ The vertical scale for each spectrum is normalized to the peak intensity of the $D_{1}$ band. 
near the time origin led to underestimation in the least squares fitting using Eqs. (4a) and (4b). In addition, this may also be responsible for a small peak shift of the $D_{1}$ bands between the two experiments.

Ultrafast IC from $S_{2}$ via the $S_{2} / S_{1}$ CI creates a vibrationally hot $S_{1}$ state, and $C_{S_{1}}(E)$ shown in Fig. 7(b) corresponds to the photoelectron spectrum for this state. However, the $S_{1}$ photoelectron spectrum can be obtained without relying on the least squares fitting: integration of the observed PKEDs from -162 to -107 fs provides a "time-averaged" $S_{1}$ photoelectron spectrum, because the photoionization signal from $S_{2}$ is negligible for the corresponding delay times, as can be seen in Fig. 6. The result is presented as a function of eBE in Fig. 9 and is compared with the $S_{1}$ spectrum obtained from the $3 \omega+6 \omega$ experiment. Again, it is clear that the results are consistent with each other, although they deviate slightly at eBEs higher than $11 \mathrm{eV}$. The band at eBE $=\sim 10.5 \mathrm{eV}$ is due to the $D_{0} \leftarrow S_{1}$ photoionization process. The lowest eBE (adiabatic ionization energy for $D_{0}$ ) is $9.288 \mathrm{eV} ;{ }^{16}$ however, the observed peak position for $D_{0}$ in Fig. 9 is approximately $10.5 \mathrm{eV}$. The energy difference between $S_{2}$ and $S_{1}, 0.86 \mathrm{eV},{ }^{22,23}$ is transformed into the $S_{1}$ vibrational energy upon IC, and the vibrational energy is approximately conserved upon $D_{0} \leftarrow S_{1}$ photoionization, which increases the apparent eBE. The observed shift of $\sim 1.2 \mathrm{eV}$ is even larger than $0.86 \mathrm{eV}$, which is ascribed to the difference between the PESs of $S_{1}$ and $D_{0}$.

Lastly, we focus on the PKE dependence of the photoelectron angular anisotropy for the $D_{0} \leftarrow S_{1}$ photoionization process. Our successive studies using $4 \omega, 5 \omega$, and $6 \omega$ probe pulses enabled us to measure $\beta_{2}(E)$ for the $D_{0} \leftarrow S_{1}$ process over a wide energy range of $0-4 \mathrm{eV}$, as presented in Fig. 10. The $\beta_{2}(E)$ values were obtained in the PKE regions where the photoelectron bands corresponding to $D_{0} \leftarrow S_{1}$ photoionization were observed: $0.2-1.0,1.5-2.5$, and $3.0-4.0 \mathrm{eV}$ for the $4 \omega, 5 \omega$, and $6 \omega$ probe pulses, respectively. The $\beta_{2}$ values were time-averaged over the pump-probe delays

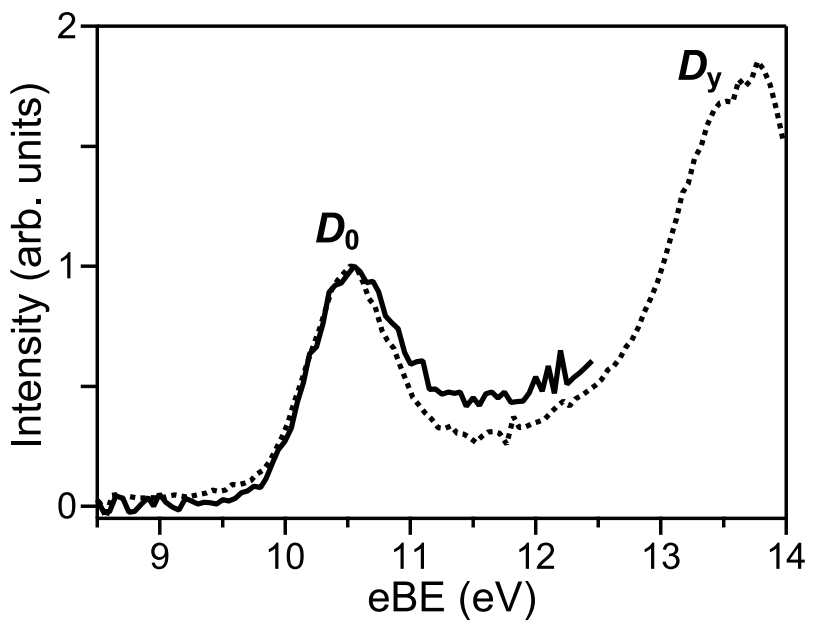

FIG. 9. Comparison of the photoelectron spectra for vibrationally hot $S_{1}$ obtained by $5 \omega$ (solid line) and $6 \omega$ (dotted line) probe pulses. The solid line is obtained by integration of the PKEDs presented in Fig. 4(b) from -162 to $-107 \mathrm{fs}$, while the dotted line is obtained by the integration of the PKEDs presented in Fig. 6(a) of the preceding paper from 108 to $168 \mathrm{fs}^{30}$

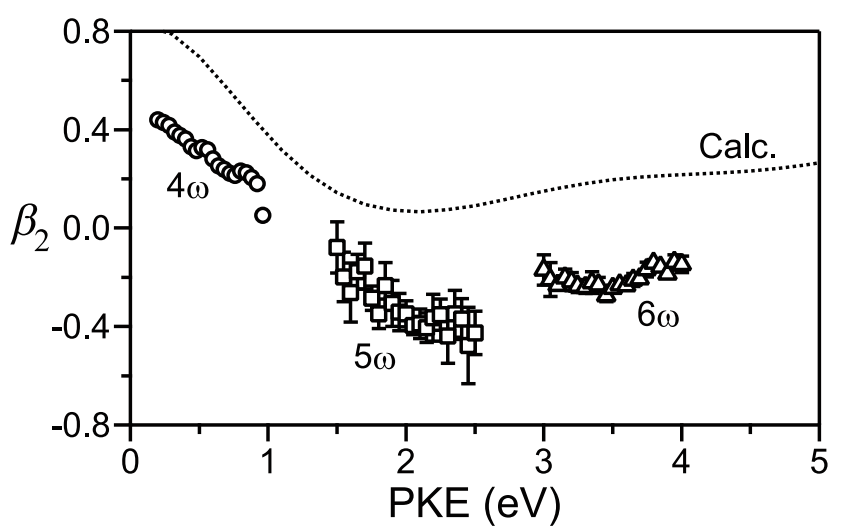

FIG. 10. $\beta_{2}(E)$ for $D_{0} \leftarrow S_{1}$ photoionization measured by $4 \omega$ (circles), $5 \omega$ (squares), and $6 \omega$ (triangles) probe pulses. The pump pulse was $3 \omega$ for each experiment. The experimental data for a $4 \omega$ probe were taken from our previous study, ${ }^{26}$ while those for $5 \omega$ and $6 \omega$ probes were obtained in the present and preceding studies, ${ }^{30}$ respectively. The $\beta_{2}$ values were time-averaged over the pump-probe delays of 103-393, 107-162, and 108-168, fs for $3 \omega-4 \omega$, $3 \omega-5 \omega$, and $3 \omega-6 \omega$ experiments, respectively. Our theoretical prediction from the previous study ${ }^{24}$ is shown with a dotted line.

of 103-393, 107-162, and $108-168$ fs for the $3 \omega-4 \omega$, $3 \omega-5 \omega$, and $3 \omega-6 \omega$ experiments, respectively.

As shown in Fig. 10, $\beta_{2}$ is around 0.4 near the threshold and exhibits a strong negative dependence on PKE from 0 to $2 \mathrm{eV}$ : the dependence is approximately linear with a slope of around $-0.4 \mathrm{eV}^{-1}$. The $\beta_{2}$ value reaches around -0.4 at $2 \mathrm{eV}$ and then gradually increases with PKE. In a previous theoretical study of the photoelectron angular anisotropy for pyrazine, ${ }^{24}$ we calculated $\beta_{2}(E)$ for the $D_{0} \leftarrow S_{1}$ process using the continuum multiple scattering $\mathrm{X} \alpha(\mathrm{CMX} \alpha)$ approximation. In the present $\left(1+1^{\prime}\right)$ REMPI scheme, the molecular axis is aligned by a $3 \omega$ fs pulse via the $y$ transition dipole $\left(S_{2},{ }^{1} B_{2 u}\right)$, and $S_{1}$ is created from $S_{2}$ by ultrafast IC ( $\sim 22$ fs) without changing the molecular axis alignment, which was taken into account in the calculation. Small variations in the molecular axis alignment due to rotational dephasing were neglected in the calculation since the observation time window is narrow ( $<200 \mathrm{fs}$ ), and the calculation was performed with both $S_{0}$ and $S_{1}$ equilibrium geometries. Further details regarding the calculations may be found in the literature. ${ }^{24}$

The $\beta_{2}(E)$ calculated for $D_{0} \leftarrow S_{1}$ photoionization using the $S_{1}$ equilibrium geometry is presented as the dotted line in Fig. 10. Our theoretical prediction qualitatively reproduces the observed PKE dependence of $\beta_{2}$ : the observed minimum for $\beta_{2}(E)$ is around $2 \mathrm{eV}$, which is in excellent agreement with our prediction. A partial wave analysis carried out in the previous study $^{24}$ showed that the pronounced variation of $\beta_{2}(E)$ is ascribed to a shape resonance, in which a photoelectron is temporarily trapped by a potential barrier due to a centrifugal force. A similar significant variation of $\beta_{2}(E)$ due to a shape resonance has recently been reported by Staniforth et al., ${ }^{25}$ in which the $S_{1}\left(\pi \pi^{*}\right)$ states of difluorobenzene isomers with ortho, meta, and para substitutions were ionized by onephoton ionizations using nanosecond UV and VUV pulses. They measured $\beta_{2}(E)$ in the PKE region from 0 to $3.5 \mathrm{eV}$ and found that for each isomer, $\beta_{2}(E)$ varied strongly due to a shape resonance: $\beta_{2}(E)$ rapidly decreases with increasing 
PKE from the ionization threshold and then starts to increase at PKEs of less than $1 \mathrm{eV}$, which was qualitatively reproduced by their $\mathrm{CMX} \alpha$ calculations.

\section{CONCLUSION}

We studied the ultrafast electronic dynamics of pyrazine using TRPEI with $3 \omega$ and $5 \omega$ femtosecond pulses. Previously, we performed TRPEI of this molecule using a VUV freeelectron laser (Spring-8 Compact SASE Source, SCSS) synchronized with a tunable femtosecond UV laser, ${ }^{27}$ in which the wavelengths of pump and probe pulses were, respectively, set at 260 and $161 \mathrm{~nm}$. The $161-\mathrm{nm}(7.7 \mathrm{eV})$ VUV pulse should have created $S_{4}$ and low-lying Rydberg states; however, the low repetition rate $(10 \mathrm{~Hz})$ of the SCSS and the limited beam time prevented us from carrying out a detailed study on these states. The present study using $3 \omega$ and $5 \omega$ generated from our $1 \mathrm{kHz}$ light source enabled direct observation of the $S_{4}$ and ${ }^{1} R(3 s),{ }^{1} R\left(3 p_{z}\right)$, and ${ }^{1} R\left(3 p_{y}\right)$ Rydberg states with a high time-resolution of $27 \mathrm{fs}$. Time-energy mapping of $\sigma(E, t)$ and $\beta_{2}(E, t)$ enabled us to discern complex electronic dynamics in the VUV region, where a large number of electronic states exist.

Using a $3 \omega$-pump and $5 \omega$-probe pulse sequence, we investigated ultrafast $S_{2}\left(\pi \pi^{*}\right) \rightarrow S_{1}\left(n \pi^{*}\right)$ IC dynamics. While the strong signal due to the $5 \omega$-pump and $3 \omega$-probe pulse sequence interfered with observation of the short-lived $S_{2}$ state, we were able to successfully extract the photoionization signal (decay associated spectrum) from $S_{2}$, which is consistent with the result obtained by TRPEI using $3 \omega$-pump and $6 \omega$ probe pulses. The PKE dependence of the photoelectron angular anisotropy for the $D_{0} \leftarrow S_{1}$ photoionization process was found to be consistent with our previous theoretical calculations. $^{24}$

As demonstrated in the preceding ${ }^{30}$ and present papers, a fs-VUV laser is a highly useful light source not only for studying the ultrafast electronic dynamics of highervalence and Rydberg states but also for observing the entire photophysical and photochemical processes using TRPES or TRPEI. Whenever it is possible, it is advantageous to set the probe wavelength to be non-resonant with any strong photoabsorption band of a target molecule. In this sense, wavelength tunability of the probe laser is useful. In our previous study, ${ }^{28}$ we employed collinear filamentation fourwave mixing using a tunable near-infrared light from a non- collinear optical parametric amplifier and the 2nd harmonic of a Ti:sapphire laser. Ghotbi et al..$^{29}$ have also developed tunable fs-VUV pulses in the wavelength range from 151 to $146 \mathrm{~nm}(8.2-8.5 \mathrm{eV})$ using non-collinear four-wave mixing. On the other hand, the key advantage of the light source with fixed photon energies employed in the preceding ${ }^{30}$ and present paper is its simplicity and ease of implementation.

${ }^{1}$ M. Stener, P. Decleva, D. M. P. Holland, and D. A. Shaw, J. Phys. B: At., Mol. Opt. 44, 075203 (2011).

${ }^{2}$ A. Bolovinos, P. Tsekeris, J. Philis, E. Pantos, and G. Andritsopoulos, J. Mol. Spectrosc. 103, 240 (1984).

${ }^{3}$ T. Suzuki, Annu. Rev. Phys. Chem. 57, 555 (2006).

${ }^{4}$ T. Horio, R. Spesyvtsev, and T. Suzuki, Opt. Express 21, 22423 (2013).

${ }^{5}$ T. Horio, R. Spesyvtsev, and T. Suzuki, Opt. Lett. 39, 6021 (2014).

${ }^{6}$ R. Spesyvtsev, T. Horio, Y.-I. Suzuki, and T. Suzuki, J. Chem. Phys. 143, $014302(2015)$.

${ }^{7}$ G. A. Garcia, L. Nahon, and I. Powis, Rev. Sci. Instrum. 75, 4989 (2004).

${ }^{8}$ M. Hackmeyer and J. L. Whitten, J. Chem. Phys. 54, 3739 (1971).

${ }^{9}$ I. C. Walker and M. H. Palmer, Chem. Phys. 153, 169 (1991).

${ }^{10}$ M. P. Fülscher and B. O. Roos, Theor. Chim. Acta 87, 403 (1994).

${ }^{11}$ J. E. Del Bene, J. D. Watts, and R. J. Bartlett, J. Chem. Phys. 106, 6051 (1997).

${ }^{12}$ W. von Niessen, W. P. Kraemer, and G. H. F. Diercksen, Chem. Phys. 41, 113 (1979).

${ }^{13}$ J. V. Ortiz and V. G. Zakrzewski, J. Chem. Phys. 105, 2762 (1996).

${ }^{14}$ D. M. P. Holland, A. W. Potts, L. Karlsson, M. Stener, and P. Decleva, Chem. Phys. 390, 25 (2011).

${ }^{15}$ C. Fridh, L. Åsbrink, B. Ö. Jonsson, and E. Lindholm, Int. J. Mass Spectrom. Ion Phys. 8, 101 (1972).

${ }^{16}$ M. Oku, Y. Hou, X. Xing, B. Reed, H. Xu, C. Chang, C. Y. Ng, K. Nishizawa, K. Ohshimo, and T. Suzuki, J. Phys. Chem. A 112, 2293 (2008).

${ }^{17}$ R. E. Turner, V. Vaida, C. A. Molini, J. O. Berg, and D. H. Parker, Chem. Phys. 28, 47 (1978).

${ }^{18}$ C. F. Dion and E. R. Bernstein, J. Chem. Phys. 103, 4907 (1995).

${ }^{19}$ J. K. Song, M. Tsubouchi, and T. Suzuki, J. Chem. Phys. 115, 8810 (2001).

${ }^{20}$ Y.-I. Suzuki, T. Fuji, T. Horio, and T. Suzuki, J. Chem. Phys. 132, 174302 (2010).

${ }^{21}$ C. Woywod, W. Domcke, A. L. Sobolewski, and H. J. Werner, J. Chem. Phys. 100, 1400 (1994).

${ }^{22}$ Y. Udagawa, M. Ito, and I. Suzuka, Chem. Phys. 46, 237 (1980).

${ }^{23}$ I. Yamazaki, T. Murao, T. Yamanaka, and K. Yoshihara, Faraday Discuss. 75, 395 (1983).

${ }^{24}$ Y.-I. Suzuki and T. Suzuki, J. Chem. Phys. 137, 194314 (2012).

${ }^{25}$ M. Staniforth, S. Daly, K. L. Reid, and I. Powis, J. Chem. Phys. 139, 064304 (2013).

${ }^{26}$ T. Horio, T. Fuji, Y.-I. Suzuki, and T. Suzuki, J. Am. Chem. Soc. 131, 10392 (2009).

${ }^{27}$ S. Y. Liu, Y. Ogi, T. Fuji, K. Nishizawa, T. Horio, T. Mizuno, H. Kohguchi, M. Nagasono, T. Togashi, K. Tono, M. Yabashi, Y. Senba, H. Ohashi, H. Kimura, T. Ishikawa, and T. Suzuki, Phys. Rev. A 81, 031403 (2010).

${ }^{28}$ P. Zuo, T. Fuji, and T. Suzuki, Opt. Express 18, 16183 (2010).

${ }^{29}$ M. Ghotbi, P. Trabs, M. Beutler, and F. Noack, Opt. Lett. 38, 486 (2013).

${ }^{30}$ T. Horio, R. Spesyvtsev, K. Nagashima, R. A. Ingle, Y.-I. Suzuki, and T. Suzuki, J. Chem. Phys. 145, 044306 (2016). 